\title{
Análise de políticas, politicas de saúde e a Saúde Coletiva
}

| ${ }^{1}$ Jeni Vaitsman, ${ }^{2}$ José Mendes Ribeiro, ${ }^{3}$ Lenaura de Vasconcelos Costa Lobato I

Resumo: O artigo analisa o desenvolvimento da Saúde Coletiva no Brasil como um campo que se estruturou com base em ao menos três princípios similares aos das policy sciences definidas nos Estados Unidos por H. Laswell na década de 1950: multidisciplinaridade, resolução de problemas e normatividade. A partir daí, a ideia de uma ciência aplicada à tomada de decisão para as políticas públicas difundiu-se sob o nome de análise de políticas. Enquanto nos Estados Unidos a normatividade foi vista pelos cientistas políticos como incompatível com a ciência, no Brasil esta convergência esteve na base da institucionalização da Saúde Coletiva como um campo acadêmico interdisciplinar e de intervenção nas políticas de saúde a partir dos anos 70 , quando o movimento pela reforma na saúde avançou sustentado em várias frentes, como academia, governo, sindicatos e outros movimentos sociais. Com a consolidação democrática e as mudanças de porte empreendidas no setor saúde, a incorporação de conhecimento científico às decisões em políticas de saúde também ocorreu no Brasil, destacando-se a produção da Saúde Coletiva reunindo ação política e conhecimento. Aborda-se, no desenvolvimento do campo da Saúde Coletiva: por um lado, o apoio do Ministério da Saúde a estudos orientados à política e o da Abrasco, no reconhecimento do caráter multidisciplinar e normativo da Saúde Coletiva. Por outro, na esfera da acreditação acadêmica, as disputas epistêmicas e a competição por recursos financeiros com o predomínio das formas de legitimação baseados nos critérios da biomedicina que vêm colocando em xeque os princípios normativos, multidisciplinares e orientados às políticas que haviam originado o campo.

> Palavras-chave: Saúde Coletiva; análise de políticas; políticas de saúde.

\author{
1 Doutora em Sociologia, \\ pesquisadora titular do \\ Departamento de Ciências \\ Sociais, Escola Nacional de \\ Saúde Pública, Fundação \\ Oswaldo Cruz (ENSP-Fiocruz). \\ Endereço eletrônico: vaitsman@ \\ uol.com.br \\ ${ }^{2}$ Departamento de Ciências \\ Sociais, ENSP-Fiocruz. Endereço \\ eletrônico: ribeiro@ensp. \\ fioruz.br \\ ${ }^{3}$ Programa de Estudos \\ Pós-Graduados em Política \\ Social. Universidade Federal \\ Fluminense. Endereço \\ eletrônico: Iv.lobato@uol. \\ com.br
}




\section{Introdução}

Como parte do desenvolvimento das ciências sociais e da atuação do Estado sobre os problemas sociais, um campo de conhecimento sobre as políticas públicas começou a se desenvolver nas primeiras décadas do século XX. Com a defesa da aplicação de princípios científicos ao estudo da política pública, a pesquisa aplicada passou a ser vista como necessária para se compreender as implicações políticas de fenômenos econômicos e psicológicos. (McCOOL, 1995; WILDAWSKY, 1997; HOWLETT; LAYCOCK, 2005; FARR et al., 2006).

Nos anos de 1950, a ideia de uma "ciência das políticas públicas" (policy science) será enunciada por Laswell (1951), que identifica o surgimento de uma orientação multidisciplinar aplicada às políticas públicas. Esta orientação teria, segundo o autor, duas direçôes: uma ciência sobre as políticas públicas, baseada no uso de métodos científicos de investigação para conhecimento sobre o processo das políticas (policy process), e uma ciência para as políticas, voltada às necessidades de inteligência do processo das políticas, à melhoria do conteúdo da informação e das interpretações para os tomadores de decisão.

A noção de ciência das políticas públicas definida por Laswell possui diferenças em relação às abordagens então correntes da Ciência Política, podendo-se destacar: a) multidisciplinaridade: implicaria ultrapassar o estudo das instituiçôes e estruturas políticas, incorporando as abordagens de campos como a sociologia, a economia e o direito; b) resolução de problemas: deveria voltar-se à resolução de problemas reais, deixando de lado os debates puramente acadêmicos; c) normatividade: não deveria se esconder sob o véu da "objetividade científica", mas reconhecer o papel dos valores no estudo das ações governamentais. Os analistas deveriam dizer claramente a melhor opção entre várias alternativas. E no contexto da Guerra Fria, os valores defendidos por Laswell eram os da democracia liberal, assumindo a ciência das políticas públicas como "ciência das políticas públicas da democracia”.

Laswell foi muito criticado pelos cientistas políticos da época, sobretudo pelo paradoxo de visão de uma "ciência normativa", que entrava em contradição com a definição positiva de ciência, centrada na objetividade e afastamento em relação a valores. Mesmo criticada, essa proposta, de forma modificada, acabou se difundindo sobretudo nos EUA, não mais como "ciência das políticas públicas", mas como "análise de políticas públicas" (policy analysis) referindo-se a estudos, 
pesquisas e análises dirigidos à tomada de decisão governamental. Esse processo tomou impulso com o crescimento do financiamento público de programas sociais e econômicos no pós-guerra europeu, com a expansão do domínio norte-americano e, na década de 60, com o movimento pelos direitos civis e as políticas contra a pobreza, quando se fez necessário reunir conhecimento setorial e gestão pública.

Sob o nome de "análise de políticas", associada a disciplinas acadêmicas como a Ciência Política ou a Administração, ou ainda como um subcampo da disciplina Política Pública, essa abordagem se desenvolveu em diferentes contextos nacionais com características específicas. Em seu paradigma clássico e principalmente a partir da literatura norte-americana, veio designar uma modalidade específica de análise orientada para a resolução de problemas de políticas públicas, baseado em uma metodologia específica, que depois também se diversificou. Essa metodologia de análise de políticas, considerada por Géva-May (2002) como o eixo básico do campo, difundida pela literatura da área e ensinada em cursos de políticas públicas nos EUA, consiste em uma abordagem operacional que passa pelos estágios de definição do problema; seleção de alternativas de políticas; argumentação, seleção de uma política; desenho de implementação e avaliação (WEIMER, 1989; PATTON; SAWICKI, 1993; BARDACH, 1996). No Brasil, embora a análise de políticas seja exercida na prática, como art and craft (WILDAWSKY, 1979), não é definida por uma metodologia própria, ainda que metodologias de planejamento e análises sobre objetos de políticas públicas passem por algumas das etapas do paradigma clássico norte-americano.

No Brasil não se estabeleceu a diferença conceitual entre análises sobre o processo das políticas e para o processo das políticas (LASSWELL, 1951). Em alguns contextos nacionais, essa distinção originou a separação entre uma área de estudos acadêmica, seguindo os paradigmas das ciências humanas e sociais, e outra que, embora exercida também a partir de expertise adquirida em instituiçôes acadêmicas, é aplicada visando às necessidades dos tomadores de decisão, ou para influenciar o curso das políticas (WEIMER, 1989; PATTON; SAWICKI, 1993; BARDACH, 1996; DOBUZINSKIS et al., 2007). Aqui, o termo "análise de políticas" costuma ser empregado indistintamente para se referir a vários tipos de produção de conhecimento sobre políticas públicas - estudos, pesquisas acadêmicas, diagnósticos, análises - inclusive os que são realizados como consultorias e assessorias a governos. 
Andrews (2005) argumenta que as "ciências das políticas públicas" se desdobraram em duas vertentes teóricas e várias correntes analíticas. Por um lado, a desenvolvida a partir dos estudos de Arrow que inspiraram as teorias da escolha pública (public choice) e da escolha racional na Ciência Política. Essas abordagens teriam aberto caminho para aplicação dos métodos econômicos na análise política que nas décadas de 80 e 90 estiveram no cerne da primeira geração de Reformas do Estado, marcadas por maior orientação aos mercados; em ondas seguintes, teriam se associado ao modelo de New Public Management, orientadas a resultados mensuráveis. Outra vertente englobaria as abordagens teóricas que priorizam métodos interpretativos e os procedimentos participativos na formulação e implementação de políticas públicas.

Vaitsman et al. (2013) apontam que no Brasil a emergência da análise de políticas enquanto uma atividade levou à profissionalização da atividade em diferentes esferas governamentais e societárias, comportando especialidades diversas, ainda que não por meio da estruturação de uma profissão formal de analista de políticas. Em comum a estas atividades estão o caráter multidisciplinar e a orientação às políticas governamentais. Atividades de análise direcionadas às decisões em políticas públicas tornaram-se proeminentes no país em vários ciclos de desenvolvimento político e econômico, especialmente sob forte demanda governamental, expandindo-se com a redemocratização do país no pós-1985. Nessas condições, a profissionalização da análise de políticas se estendeu das estruturas de governo para as arenas políticas povoadas pelo ativismo societário. Desenvolveu-se no bojo da expansão da burocracia de Estado, das elites técnicas e intelectuais e da proliferação de comunidades civis envolvidas com a formulação de políticas em diferentes contextos históricos (Vaitsman et al., 2013).

Argumentamos que a Saúde Coletiva, desenvolvida pela articulação entre produção de conhecimento e intervenção sobre as políticas de saúde, constitui campo de análise de políticas com princípios similares aos da ciência das políticas públicas proposta por Laswell - multidisciplinaridade, resolução de problemas e normatividade. Consiste no que Andrews (2005) tratou como a vertente associada aos procedimentos participativos no desenvolvimento das "ciências das políticas públicas".

Enquanto nos EUA a normatividade foi vista por muitos cientistas políticos como incompatível com a ciência, no Brasil esta convergência está na origem da Saúde Coletiva como um campo de conhecimento e de intervenção sobre as 
políticas de saúde. Teve origem na década de 1970, a partir da crítica à Saúde

Pública feita por acadêmicos de esquerda nos departamentos de medicina social em algumas universidades públicas. Inicialmente fortemente teórica, tornou-se um projeto político e técnico com base no materialismo histórico, associando democracia a uma radical transformação do sistema de saúde. Um projeto que nos anos 80 se desenvolveu como um amplo movimento social pela democracia e a proposta de um sistema público e universal de saúde (TEIXEIRA, 1985; NUNES, 1994; ESCOREL, 1998; AROUCA, 2003). O sucesso do movimento sanitário reformador concretizou-se com a incorporação da proposta de reforma na Constituição de 1988 e a criação do Sistema Único de Saúde (SUS).

Neste artigo, procuramos discutir que essa especificidade da Saúde Coletiva, de campo de análise de políticas baseado na multidisciplinaridade, resolução de problemas e normatividade, vem se alterando, com progressiva diferenciação entre a produção típica de "análise de políticas" e a produção acadêmica stricto sensu. Por um lado, tem-se a "análise de políticas", como um tipo de conhecimento utilizado para apoiar ou influenciar dimensōes do processo das políticas, cada vez mais utilizado pelos governos, principalmente o federal. Por outro, o campo acadêmico, cada vez mais orientado pela biomedicina e epidemiologia que, ao reivindicar legitimação científica à Saúde Coletiva, coloca em xeque seus princípios normativos, multidisciplinares e de orientação às políticas. Um razoável equilíbrio entre essas duas áreas vem sendo até agora em grande parte garantido pela Associação Brasileira de Saúde Coletiva (Abrasco) que, atuando concomitantemente nas frentes acadêmica, técnica e política, mantém a unidade do campo, apesar da crescente especialização e conflito em seu interior.

Discutimos esse movimento de diferenciação a partir da análise da trajetória histórica da Saúde Coletiva e do financiamento recente de estudos e pesquisas pelo Ministério da Saúde. Diferenciação entre, por um lado, a área da acreditação acadêmica, apoiada pelas agências federais de fomento a ensino e pesquisa (CAPES e CNPq), que também se diferenciou internamente com a hierarquização da produção acadêmica, dos meios de divulgação científica e a competição por recursos financeiros sob forma de bolsas e financiamentos a pesquisa, congressos e publicações. Por outro, a esfera da produção de análise de políticas, orientada à tomada de decisão, que criou novas oportunidades a grupos de pesquisa e pesquisadores, paralelamente ao sistema acadêmico. 


\section{O desenvolvimento do campo da Saúde Coletiva}

A institucionalização em estruturas acadêmicas, políticas e nas políticas de saúde é a marca distintiva da Saúde Coletiva brasileira. Congregando disciplinas das ciências sociais e da saúde, a Saúde Coletiva emergiu na década de 1970, quando profissionais de saúde, acadêmicos, partidos políticos e movimentos sociais criaram instituições de ação política e de produção científica cuja base normativa era o combate à ditadura militar e a defesa da democracia e da saúde como direito universal. A imbricação entre produção de conhecimento e militância política, além de influenciar a agenda e as alternativas de políticas, gerou um campo de conhecimento institucionalizado na estrutura oficial da pós-graduação brasileira. Esta comunidade de especialistas atuou decisivamente a partir de suas posições em universidades, na administração pública e por meio de seus vínculos com organizações e movimentos societários ao longo de redemocratização do país (COSTA, 2013). A contínua produção de análises de políticas governamentais e de propostas reformadoras está na origem da formação da Saúde Coletiva junto à agenda da reforma sanitária dos anos 1980. O termo "Saúde Coletiva" não encontrava paralelo em nomenclaturas de especialidades na época. As denominações conhecidas eram as de saúde pública, medicina social e medicina preventiva, com departamentos em faculdades de medicina e de enfermagem. Com a nova denominação buscava-se marcar diferenças com áreas médicas tradicionais e afirmar referências e paradigmas como a epidemiologia social e o foco em mudanças sociais impulsionadas por políticas setoriais.

As tradições institucionais de matrizes biomédicas da saúde pública brasileira convergiram com as novas ênfases nos contextos socioeconômicos. Construía-se um campo híbrido, congregando tanto a produção acadêmica tradicional da biomedicina, baseada em evidências e com a participação de estudos experimentais, quanto novas formas de produção baseadas em abordagens históricas e sociais orientadas à formulação e à implementação de políticas. Nesse momento fundador de um novo campo reunindo diferentes áreas de conhecimento (políticas públicas, planejamento e gestão, ciências sociais e humanas, epidemiologia e biomedicina), a produção acadêmica se institucionalizou de modo imbricado à própria reforma do sistema de saúde. Como parte da vertente crítica, desenvolveram-se abordagens das "ciências das políticas públicas" fundadas em normatividade, resolutividade e multidisciplinaridade. 
Periódicos científicos foram formados ou fortalecidos a partir de instituições

universitárias e associações. O perfil das publicaçōes nessas revistas e em livros refletia a pluralidade de um campo compartilhado entre as áreas da biomedicina associada à epidemiologia e a das ciências humanas e os estudos de políticas.

Uma das principais expressões do desenvolvimento e das características do campo da Saúde Coletiva é a Abrasco, criada em 1979 com a participação de técnicos, profissionais, alunos e professores da área. A Abrasco deveria congregar os interesses dos diferentes cursos de pós-graduação da área, com atuação voltada para o ensino e a formação dos profissionais de saúde. Contudo, o contexto político do país durante a década de 1980 e a militância de seus dirigentes acabou lhe dando papel central nas lutas pela democracia e pela reforma universalista do sistema de saúde brasileiro. Tornou-se um espaço de articulação e convivência entre duas lógicas, a científica e a política. Uma combinação de aspectos acadêmicos e normativos. Os primeiros, relativos à institucionalização de uma nova área de conhecimento na estrutura do sistema nacional de pós-graduação; os segundos, relativos à intervenção na política setorial.

Desde sua criação, a Abrasco desempenhou papel tanto nos debates, na produção de análises e de articulação política no setor saúde, quanto na legitimação científica de um campo multidisciplinar de conhecimento, cujos participantes eram ao mesmo tempo acadêmicos, profissionais de saúde e militantes da reforma do sistema de saúde. Todos os seus presidentes têm sido reconhecidos pesquisadores de instituições científicas e vários deles assumiram em seguida altos cargos como gestores da política de saúde. Esse papel singular foi alcançado pela articulação das esferas da política, das políticas públicas de saúde e da academia, através de ações e posicionamentos políticos e produção de documentos setoriais com a chancela de sua representatividade acadêmica. Atuou em diferentes fóruns e organismos científicos nacionais e internacionais e organizou congressos e conferências científicas. Interveio junto aos órgãos de regulação e fomento na definição de critérios de financiamento de bolsas para os pesquisadores da área de Saúde Coletiva. Isso garantiu à Abrasco, e à área de Saúde Coletiva, legitimidade científica conjugada à orientação à política de caráter multidisciplinar e normativo.

O desenvolvimento desse campo híbrido, com fronteiras elásticas entre ciência e política, não poderia ser imune a conflitos. A interdisciplinaridade da 
Saúde Coletiva acabou produzindo disputas epistêmicas entre suas diversas áreas de conhecimento, com repercussões na hierarquização de programas de pósgraduação e no financiamento de bolsas e de pesquisas. Ao mesmo tempo, ao longo de três décadas, a gestão pública na saúde se profissionalizou, demandando conhecimento na forma de "análise de políticas".

\section{Os conflitos na área acadêmica e a Saúde Coletiva}

$\mathrm{Na}$ área acadêmica, com a progressiva internacionalização da pós-graduação brasileira, o aumento da competição por registros de patentes e publicações em periódicos de maior impacto e projeção, disputas epistêmicas por legitimidade científica entre as ciências biomédicas e as ciências sociais e humanas vêm desafiando as características originais do campo fundado na interdisciplinaridade. $\mathrm{Na}$ biomedicina predominam os métodos típicos dos estudos experimentais e observacionais com evidências baseadas em amostras ou passíveis de reprodução. $\mathrm{Na}$ multidisciplinaridade predominam teorias, conceitos e métodos clássicos das ciências humanas, e os estudos baseados em evidência frequentemente perseguem objetivos normativos em termos de policy orientation. Nos comitês e órgãos reguladores acadêmicos do campo das ciências da saúde, a legitimidade e as disputas acadêmicas têm sido amplamente favoráveis às áreas biomédica e epidemiológica, que conseguiram definir os critérios de cientificidade e, consequentemente, as regras de acesso aos mecanismos de apoio e financiamento à pesquisa, em prejuízo da concepção interdisciplinar da Saúde Coletiva.

A pós-graduação brasileira se expandiu enormemente desde 1976, quando passou de 699 cursos para 4.101 em 2009, um crescimento de 370,3\% no número dos cursos de mestrado e $685,6 \%$ nos de doutorado no período. O número de docentes na pós-graduação aumentou de 13.349 em 1987 a 57.270 em 2009 (BRASIL, 2010). Na área da Saúde Coletiva, no final da década de 90, existiam 33 programas de pós-graduação (NUNES, 2005) e em 2011 esse número chegou a 48 (HOLLANDA et al., 2011). A expansão da educação superior e a busca por profissionalização, qualificação e maiores salários por meio de cursos de pósgraduação produziram maior especialização e competição por recursos sob a forma de bolsas e financiamentos de pesquisas.

As duas agências federais responsáveis pelo apoio e regulação do ensino de pósgraduação e o fomento à pesquisa - CAPES e $\mathrm{CNPq}^{1}$ - classificam da mesma forma 
os programas de pós-graduação, os quais se vinculam a uma Área de Conhecimento, que por sua vez se vincula a uma Grande Área. A área de conhecimento da Saúde Coletiva pertence à Grande Área de Ciências da Saúde, que inclui também as áreas de Medicina, Odontologia, Farmácia, Enfermagem e Educação Física.

Os programas de pós-graduação da área de Saúde Coletiva são avaliados por uma comissão composta por membros das instituições associadas. Os critérios de avaliação são definidos em consonância com os investigadores da área, que no caso da Saúde Coletiva, são representados pela sua associação, a Abrasco. A publicação em periódicos classificados segundo impacto constitui o principal critério da avaliação. Os maiores conflitos se dão em torno dos critérios de classificação dos periódicos e seu índice de impacto, pois é a partir daí que se estabelece uma hierarquia entre periódicos, pesquisadores e programas. No caso da Saúde Coletiva, de caráter multidisciplinar, as várias disciplinas que a compõem têm diferentes tradições de pesquisa, de processos de produção de conhecimento e de padrões de autoria (LUZ, 2005; LOYOLA, 2008; CAMARGO, 2010; COSTA, 2012). A expansão da pós-graduação acirrou a competição por status, espaços editoriais, poder institucional e financiamento, principalmente entre as ciências médicas e a epidemiologia, por um lado, e as ciências sociais e humanas e o planejamento, por outro.

Os argumentos em torno das diferenças de processos de produção de pesquisas vêm se tonando mais explícitos. Camargo Jr. et al. (2010) analisaram o perfil distinto das publicaçôes segundo estes dois grandes campos da Saúde Coletiva e mostraram que a característica reflexiva do modo de produção das ciências humanas se diferencia do modo epidemiológico, o qual vem conseguindo alcançar a condição paradigmática. Nesta direção, Andrea Loyola, pesquisadora da área da Saúde Coletiva e ex-presidente da Capes, vê a disciplinarização e burocratização do campo da Saúde Coletiva como o resultado da ausência de um projeto político coletivo capaz de aglutinar em uma só direção os esforços do conjunto de profissionais e de disciplinas que compõem a área (LOYOLA, 2008). $\mathrm{O}$ que procuramos mostrar aqui é que essa característica inicial do campo, sua fundação a partir do projeto político de diferentes comunidades epistêmicas, não existe mais. Pelo contrário, serve justamente de ponto de discórdia para uma geração mais jovem e submetida aos critérios de produtividade científica segundo padrões internacionais. 
Costa (2012) assinala a hegemonia do conhecimento biomédico nos periódicos de maior pontuação na Saúde Coletiva. Demonstra que os critérios vigentes de avaliação reiteram e produzem as vantagens dos pesquisadores do polo biomédico da Saúde Coletiva e que isto decorre da própria racionalidade de seu processo de produção de conhecimento. Publicações de durabilidade curta no tempo e aplicações de métodos objetivos não dependentes de reflexões conceituais vão cada vez mais conformando um padrão editorial na forma de um paradigma no sentido clássico e padronizam a produção intelectual. As repercussōes de um processo hegemônico que, como assinalamos, representa uma ruptura interna ao campo da Saúde Coletiva, já podem ser percebidas.

Essas disputas por competência se manifestam também entre as diferentes comunidades epistêmicas representadas no interior da Abrasco, e que são as mesmas que competem por legitimidade científica nas estruturas da pósgraduação. Até 1990, realizava-se um único congresso da área, o Congresso de Saúde Coletiva, congregando milhares de participantes entre pesquisadores de diferentes formações, professores, profissionais dos serviços, representantes governamentais, movimentos sociais, organizações não governamentais. Em 1990, a Comissão de Epidemiologia da Abrasco realiza seu primeiro congresso separadamente, o que já sinaliza a tendência de segmentação disciplinar. Formaram-se dois grandes grupos, dentro dos quais outras divisões ocorrem: por um lado, as áreas biomédicas - e sua expressão na forma de métodos e procedimentos em epidemiologia - e por outro, a área das ciências humanas como a economia, ciências sociais, políticas públicas, administração e planejamento. Em um processo similar ao que ocorrera com a Epidemiologia, a área de Ciências Humanas e Sociais passa a organizar seu próprio congresso e em 2010 realizou-se o primeiro congresso de Política e Planejamento da Associação.

Questôes epistêmicas deste tipo não são exclusivas do processo aqui discutido em relação à Saúde Coletiva. Há um debate internacional sobre inovação tecnológica e as mudanças no modo de produção de conhecimento e de seus mecanismos de transmissão para a sociedade, incluindo os sistemas de legitimação científica. A partir de Gibbons, Pellegrini Filho (2004) discute processos observados em áreas distintas e centros de inovação em saúde na América Latina, assinalando a transição da ciência baseada nas certezas e verdades para a validação baseada em 
critérios mais flexíveis e "socialmente distribuídos". Ou seja, a validação científica transita da certeza à incerteza e de modo mais fiel à complexidade dos problemas analisados e a seus limites.

A competição de caráter paradigmático em curso vem definindo regras de funcionamento das instituições que gravitam em torno do campo da Saúde Coletiva. Cientistas políticos institucionalistas analisaram os efeitos futuros produzidos por regras definidas em algum ponto no tempo e que se reiteram e se fortalecem a cada rodada de decisões. A estrutura e as regras do jogo que resultam de conflitos como os aqui analisados, uma vez consolidadas, tendem a afetar ou definir resultados futuros em função da institucionalização configurada a partir de um ciclo virtuoso que se reproduz. Pierson (2004) define tais processos como dependentes de trajetórias históricas (path dependence) originadas em decisões cruciais que, uma vez sujeitas a retornos crescentes e dotadas de feedback positivos, estabelecem condiçóes institucionais que acabam fazendo com que os custos para a mudança de rumos políticos se tornem progressivamente elevados para seus proponentes. Os custos de reversão de uma estrutura decisória institucional como a que vem se delineando no campo da Saúde Coletiva podem se tornar tão elevados que há o perigo de que as novas gerações de pesquisadores da área se conformem em produzir o que Kuhn (1962) tratou como atividades que apenas reproduzem a ciência normal expressa pelo paradigma dominante.

A competição por status e recursos entre pesquisadores e instituições da área junto aos órgãos federais de fomento acadêmico é impulsionada pela magnitude desses financiamentos orientados a atividades de pesquisa, desenvolvimento de pós-graduação e treinamento avançado de professores de institutos, faculdades e universidades brasileiras, que em 2008 alcançou R $\$ 2.863 .157 .129,85$ correntes, $^{2}$ sendo o orçamento executado pelo CNPq (incluindo fundos setoriais e repasses) no valor de R \$ 1.423.623.000,00 e pela CAPES no total de $\mathrm{R} \$ 1.439 .534 .129,85 .{ }^{3}$ Por outro lado, expressivo volume de recursos pode ser acessado para diferentes tipos de estudos, diagnósticos, avaliações, ou seja, para análise de políticas (em conjunto com os recursos de formação profissional) junto ao Ministério da Saúde, afora as demais instâncias do executivo estadual e municipal de saúde e de diferentes institutos no país. 


\section{Projetos de análise de políticas e as oportunidades de financiamento pelo Ministério da Saúde}

A produção de análise de políticas para a saúde envolve pesquisadores e professores com atuação em políticas públicas em inúmeras instituições acadêmicas brasileiras. Os recursos gastos pelo MS em atividades de caráter multidisciplinar geram oportunidades para a atuação destes grupos em análise e treinamento de profissionais. Isto não exclui a demanda por estudos biomédicos e epidemiológicos que estão associados à atuação do MS em termos de controle epidemiológico, prestações de serviços médicos e distribuição de medicamentos.

O MS desenvolve funções de financiamento e provisão de serviços de saúde. Sua estrutura burocrática central abrange desde os departamentos da hierarquia organizacional até os mecanismos híbridos, que incluem as relações federativas, as parcerias com entes e prestadores privados e organismos colegiados de representação da sociedade civil. Nessas arenas políticas complexas, que atravessam a estrutura burocrática do MS, desenvolve-se intensa competição por ideias e soluções e pela definição de problemas e prioridades para a política pública. Os métodos e procedimentos do campo da Saúde Coletiva interagem nestas arenas políticas em meio à competição por agendas e alternativas. Em função do próprio papel do MS na implementação das políticas de saúde, suas secretarias representam fonte relevante para os estudos multidisciplinares orientados à decisão em políticas.

A estrutura burocrática do MS fornece pistas para entender as interações com pesquisadores das ciências humanas e oportunidades de financiamento. O MS atua principalmente no financiamento de serviços, estabelecimento de normas técnicas, elaboração e coordenação de programas de saúde pública e um rol variado de funções e atividades regulatórias. Envolve, além do gabinete central do ministro, um colegiado compartilhado com organismos da sociedade civil (Conselho Nacional de Saúde), seis secretarias e um rol de fundações, autarquias, fundaçōes e empresas públicas. Em todos esses setores demandam-se informações, estudos e análises de políticas. Em termos de recursos financeiros despendidos e volume de trabalho agregado, a produção de documentos se dá em alta escala e parte relevante é de acesso livre. O MS produz e patrocina um número elevado de publicações, como séries de dados e de revistas com graus diferenciados de 
padrões acadêmicos. Em comum, esta produção está voltada principalmente para estudos e análises de políticas. Este conjunto expressa, na arena política do MS, o espaço de atuação e cooperação dos pesquisadores e grupos de pesquisa orientados a estudos multidisciplinares e a análise de políticas. O ativismo político do MS é fortalecido por meio de seus programas, redes e parcerias que expressam uma agenda sobre temas como direitos de minorias ativas, protocolos de atenção, advocacy orientada a grupos sob risco social e inovações organizacionais.

Com base nos relatórios de gestão das secretarias do MS, investigamos seus principais agregados de despesa. Os dados obtidos não são homogêneos quanto a períodos, mas permitem identificar um rol de açōes e programas relacionados, em linhas gerais, a atividades de análises de políticas e treinamento de profissionais. Adotamos critérios de classificação segundo grandes agregados, para uma análise exploratória dos gastos realizados. Esses procedimentos permitiram estimar razoavelmente, para os objetivos deste estudo, os gastos realizados para "contratação de análises, estudos e de cursos". ${ }^{4}$

As despesas foram classificadas em dois grandes grupos. Um deles, principal entre as atividades finalísticas do MS, inclui as despesas orientadas a serviços e gestão burocrática, tais como financiamento de serviços, transferências federativas, auditoria e acompanhamento burocrático. Este grupo pode incluir também despesas de treinamento, cooperação e de estudos, mas estes valores não podem ser identificados nos relatórios de gestão analisados. O outro grupo, foco principal da atividade dos pesquisadores e analistas de políticas e estudos multidisciplinares, inclui as despesas orientadas a estudos de políticas, cooperação e treinamento, tais como financiamento de estudos, análises, avaliações, recomendações e treinamento de profissionais para o SUS. Não foi possível separar com precisão as atividades orientadas ao treinamento profissional daquelas de pesquisa e consideramos para este artigo que são atividades que guardam sinergias, especialmente em se tratando das instituiçôes contratadas.

Para fins de uma referência mais geral, destacamos que o orçamento do MS aprovado para 2012 foi de $\mathrm{R} \$ 79,5$ bilhōes, dos quais 49\% previstos para gastos com assistência hospitalar e ambulatorial, principalmente transferências a estados e municípios e pagamento de prestadores privados do SUS. Para os anos que variam entre 2005 e 2009 , aqui tomados em conjunto e em valores 

não refletem o gasto total do MS, pois excluem as despesas de pessoal e se concentram nas atividades finalísticas em ações programáticas, transferências financeiras e financiamento de serviços produzidos. Para fins ilustrativos, com a ressalva de se tratar de anos distintos, embora preservando os mesmos critérios de agregação de gastos, o total de gastos das secretarias aqui estimados foi de R \$ 1.076.535.214,94 para o conjunto de atividades aqui classificadas como "estudos de políticas, cooperação e treinamento", representando 3,02\% do gasto total, como mostra a tabela 1 .

Tabela 1. Gastos do MS segundo Secretarias e consolidados de programas e ações*, Reais (valores nominais), anos diversos

\begin{tabular}{|c|c|c|c|}
\hline $\begin{array}{l}\text { Secretarias** } \\
\text { (ano) }\end{array}$ & $\begin{array}{c}\text { Serviços e gestão } \\
\text { burocrática }(\%)\end{array}$ & $\begin{array}{c}\text { Estudos de políticas, } \\
\text { cooperação e } \\
\text { treinamento }(\%)\end{array}$ & Total (\%) \\
\hline SAS (2009) & $\begin{array}{r}33.246 .072 .180,68 \\
99,9\end{array}$ & $\begin{array}{r}38.377 .079,33 \\
0,1\end{array}$ & $33.284 .449 .260,01$ \\
\hline SCTIE (2005) & $\begin{array}{r}973.771 .015,36 \\
93,9\end{array}$ & $\begin{array}{r}63.800 .000,00 \\
6,1\end{array}$ & $1.037 .571 .015,36$ \\
\hline SE (2007) & $\begin{array}{r}271.592 .868,41 \\
47,5\end{array}$ & $\begin{array}{r}299.685 .997,67 \\
52,5\end{array}$ & $571.278 .866,08$ \\
\hline SGTES (2007) & 0,0 & $\begin{array}{r}548.874 .419,69 \\
100,0\end{array}$ & $548.874 .419,69$ \\
\hline SVS (2006) & $\begin{array}{r}772.800,00 \\
0,9\end{array}$ & $\begin{array}{r}84.105 .940,00 \\
99,1\end{array}$ & $84.878 .740,00$ \\
\hline SGEP (2009) & $\begin{array}{r}35.974 .095,19 \\
46,3\end{array}$ & $\begin{array}{r}41.691 .778,25 \\
53,7\end{array}$ & $77.665 .873,44$ \\
\hline Total & $\begin{array}{r}34.528 .182 .959,64 \\
97,0\end{array}$ & $\begin{array}{r}1.076 .535 .214,94 \\
3,0\end{array}$ & $\begin{array}{r}35.604 .718 .174,58 \\
100,0\end{array}$ \\
\hline
\end{tabular}

Fonte: Relatórios de Gestão e de Atividades das Secretarias segundo o último ano disponível. *Excluídas despesas com pessoal, dívidas financeiras e similares.

** SAS (Sec. de Assistência à Saúde); SCTIE (Sec. de Ciência, Tecnologia e Insumos Estratégicos); SE (Sec. Executiva); SGETS (Sec. de Gestão do Trabalho e da Educação na Saúde); SVS (Sec. de Vigilância Sanitária); SGEP (Sec. de Gestão Estratégica e Participativa). 
Neste grupo, um total de $\mathrm{R} \$ 548.874 .419,69$ (51,0\%) corresponde à secretaria (SGTES) responsável pelo treinamento de profissionais. O segundo maior gasto - R\$299.685.997,67 (27,8\%) - corresponde à Secretaria Executiva e caracteristicamente se destina às estratégias de políticas do MS.

Lembramos que as atividades de treinamento representam área de atuação e de captação de recursos relevante para os mesmos pesquisadores e grupos que atuam em áreas multidisciplinares, embora os dados não permitam determinar em que escala esta sinergia ocorre. Igualmente na SAS, existe um conjunto de linhas de ações programáticas tradicionalmente orientadas à produção de análises e normativos de implementação de políticas típicas da saúde pública tradicional, como atenção às mulheres, crianças, adolescentes, saúde mental, idosos e portadores de deficiência. Esta secretaria realizou uma série de gastos com análise de políticas, como veremos.

A tabela 2 detalha os gastos com programas e ações para as despesas orientadas a pesquisas, análises, cooperação e treinamento, e permite analisar a composição dos recursos disponíveis para as equipes que atuam mais próximas a atividades governamentais da gestão do SUS. 

treinamento, relatórios de gestão, anos diversos, Reais nominais*

\begin{tabular}{|c|c|}
\hline MS/Secretarias & Gastos \\
\hline \multicolumn{2}{|l|}{ SGTES (2007) } \\
\hline Transf Planos Regionais Educação Perm & $84.242 .485,45$ \\
\hline Projetos Pro-Saúde & $39.833 .627,21$ \\
\hline Projetos Residência Multiprofissional & $58.786 .021,69$ \\
\hline Projetos Pesq nas Escolas Técnicas SUS & $919.765,00$ \\
\hline Projetos Formação THD/ACD & 12.036.097,00 \\
\hline PROFAE - MS; BID & $26.233 .481,23$ \\
\hline Educação Perm Qualif Profissional SUS & $322.222 .942,11$ \\
\hline Gestão Trabalho SUS & $4.600 .000,00$ \\
\hline Subtotal (a) & $548.874 .419,69$ \\
\hline \multicolumn{2}{|l|}{ SAS (2009) } \\
\hline Pol Nac Sangue Hemoderivados & $10.740 .925,00$ \\
\hline Implement Pol Atenção Saúde Mulher & $8.707 .975,36$ \\
\hline Implement Pol Atenção Saúde Mental & $856.000,00$ \\
\hline Implement Pol Atenção Saúde Adolescente Jovem & $3.865 .844,60$ \\
\hline Implement Pol Atenção Saúde Criança & 7.761.198,00 \\
\hline Implement Pol Atenção Saúde Pessoa Idosa & $4.172 .363,74$ \\
\hline Implement Pol Atenção Saúde Pessoa Deficiência & $1.025 .992,20$ \\
\hline Implement Pol Atenção Saúde Pop Penitenciária & $675.000,00$ \\
\hline Implement Pol Atenção Saúde Homem & $571.780,43$ \\
\hline Subtotal (b) & 38.377.079,33 \\
\hline \multicolumn{2}{|l|}{ SVS (2006) } \\
\hline Repasses SES capacit (Dengue) & $2.605 .940,00$ \\
\hline Diagn situacional ex-hospitais colônias & $800.000,00$ \\
\hline Vigil doenças não transm - projetos est/munic & $9.200 .000,00$ \\
\hline VIGISUS II/cp IV - transf. fortal est/munic & $56.500 .000,00$ \\
\hline VIGISUS II - capacit descentralizadas & $15.000 .000,00$ \\
\hline Subtotal (c) & $84.105 .940,00$ \\
\hline
\end{tabular}




\begin{tabular}{|c|c|}
\hline MS/Secretarias & Gastos \\
\hline \multicolumn{2}{|l|}{ SE (2007) } \\
\hline Invest qualif atenção saúde gestão SUS & $137.801 .831,07$ \\
\hline Projetos Gestão/humaniz serv saúde & $9.194 .746,22$ \\
\hline Capacit servidores pub federais & $6.521 .954,73$ \\
\hline Preserv Patrim científ cult hist saúde Brasil & $956.086,99$ \\
\hline Gestão acervos inform SUS & 2.204.781,98 \\
\hline Gestão produção editorial MS & $1.462 .007,54$ \\
\hline Gestão Banco preços saúde & $1.272 .600,00$ \\
\hline Estrut Centro Economia Saúde Ciência Tec SUS & $2.405 .100,00$ \\
\hline SIOPS & $2.477 .635,00$ \\
\hline Aperf, implem acomp de planej aval MS & 20.684.700,00 \\
\hline Qualific gestão descentralizada SUS & $38.359 .843,95$ \\
\hline Anexo $E^{* *}$ & $76.344 .710,19$ \\
\hline Subtotal (d) & 299.685.997,67 \\
\hline \multicolumn{2}{|l|}{ SGEP (2009) } \\
\hline Ampl práticas gestão participativa & 21.333.700,51 \\
\hline Ampl fortalec mobil soc defesa SUS & $6.814 .147,50$ \\
\hline Prom equidade saúde pop vulneráveis & $4.715 .762,63$ \\
\hline Atenção saúde pop quilombolas & $1.099 .371,00$ \\
\hline Monitor aval gestão SUS & $7.728 .796,61$ \\
\hline Subtotal (e) & $41.691 .778,25$ \\
\hline \multicolumn{2}{|l|}{ SCTIE (2005) } \\
\hline DECIT & $63.800 .000,00$ \\
\hline Subtotal (f) & $63.800 .000,00$ \\
\hline TOTAL MS $^{* * *}=$ subtotais $(\mathrm{a})+(\mathrm{b})+(\mathrm{c})+(\mathrm{d})+(\mathrm{e})+(\mathrm{f})$ & $1.076 .535 .214,94$ \\
\hline
\end{tabular}

* Excluídas despesas com pessoal, dívidas financeiras e similares

**Distribuição dos grupos no Anexo E (atividades em demais secretarias; estimativa a partir de planilha de execução de $15 \%$ do anexo $\mathrm{E}$

** Códigos de programas e de ações foram suprimidos; nomenclatura oficial foi preservada e resumida 
Neste grupo orientado a análise e treinamento, existem atividades típicas de análise de políticas. A MS/SE efetuou o segundo maior gasto proporcional no grupo e muitos de seus programas e ações se situam no âmbito de pesquisas e análises de políticas; parte importante desses gastos ocorre em parceria com instituições para apoio a implementadores locais de políticas prioritárias. Cerca de metade dos recursos atribuídos ao grupo aqui analisado ( $\mathrm{R}$ 548.874.419,69) estão alocados na MS/SGTES, especializada no treinamento de profissionais. Mesmo aqui, atividades como o PROFAE e o apoio a pesquisas das escolas técnicas representam projetos multidisciplinares e orientadas à análise de políticas. Na SCTIE, os recursos do DECIT destinam-se à realização de estudos, neste caso, distribuídos entre as áreas biomédicas e de ciências humanas. Alguns programas em outras secretarias (como na MS/SVS) são marcadamente orientados a análises de políticas.

No caso da MS/SAS, foram gastos $\mathrm{R} \$ 38.377 .079,33$, dos quais $\mathrm{R} \$$ 27.636.154,33 se destinaram à contratação de estudos nas áreas de análise de políticas dos clássicos programas da Saúde Coletiva e refletem parcerias das coordenações com instituições públicas prestigiosas. Envolvem atividades nas áreas de saúde mental, materno-infantil, idosos, entre outros, e costumam compor conhecidas coalizões de advocacy. Diversos outros elementos semelhantes são identificados nas demais secretarias conforme programas e ações descritos nos relatórios de gestão.

Apesar do caráter exploratório desses dados, eles revelam, em seu conjunto, grandes oportunidades de captação de recursos por fora da matriz CAPES/CNPq (também acessada para pesquisas das ciências humanas). Estas oportunidades representam fontes alternativas ao tradicional fomento acadêmico progressivamente orientado ao campo biomédico. Geram fôlego especial aos que trabalham com estudos orientados à tomada de decisão governamental e viabilizam a produção de análise de políticas, fora do modelo de acreditação acadêmica.

\section{Considerações finais}

Analisamos o desenvolvimento do campo da Saúde Coletiva à luz de distintas racionalidades, que se desenvolveram e coexistiram por um longo período sob o guarda-chuva da Abrasco junto às instituições do campo e lideranças setoriais. $\mathrm{Na}$ área acadêmica, as divisões internas ao campo parecem definitivas e com 
a especialização crescente. Segmentos tradicionais da saúde pública, como a

Epidemiologia, aproximaram-se da Biomedicina e seus padrões de produção científica, baseados na produção de evidências na linha das abordagens clínicas e experimentais. Neste segmento, o sistema de mérito, status e recursos segundo esses critérios é bem delineado. Escores baseados em publicações em periódicos que valorizam aquelas abordagens e segundo graus de impacto são indicadores fortemente socializados entre essas comunidades, em que pesem as críticas crescentes aos modelos de revisão por pares e as práticas das comunidades científicas.

Apesar do predomínio das abordagens biomédicas no sistema de acreditação acadêmica, as ciências sociais e os estudos de políticas e planejamento também conseguiram legitimidade na Saúde Coletiva e têm acesso a status e recursos. A própria Abrasco (seus congressos e publicações nas distintas áreas) passou a refletir esta diferenciação. Institucionalmente, o campo parece consolidado, repartindo-se entre uma crescente área de Epidemiologia, a qual se aproxima fortemente do modelo biomédico como disciplina acessória e de validação de inovações em diagnósticos e terapias; uma área de Ciências Sociais; e outra área orientada às políticas e planejamento de serviços de saúde. O ideário original da reforma sanitária, a crítica do sistema de saúde e a produção de soluções migram como objetos para a área de políticas e de planejamento, onde a análise de políticas e os estudos avaliativos encontram fértil campo de desenvolvimento no país.

Não é possível ainda identificar a tendência do campo da Saúde Coletiva em relação a suas origens. Tampouco é possível afirmar que há clara separação entre estudos acadêmicos e policy oriented dentro do campo. Contudo, é certo que a normatividade que pautou o crescimento da Saúde Coletiva é hoje questionada na área acadêmica. Também é fato que na área acadêmica a hegemonia hoje é da Epidemiologia, com prejuízo às ciências sociais, sejam elas aplicadas ou não, onde se concentram os estudos orientados às políticas de saúde.

O financiamento pelo MS de estudos e diferentes mecanismos de treinamento de profissionais para o desenvolvimento do SUS é fonte altamente relevante de recursos para a atuação dos pesquisadores e de grupos de estudos de caráter multidisciplinar. Muitas destas atividades são orientadas para a análise de políticas e estes recursos servem para equilibrar as oportunidades no âmbito das instituições com relação ao sistema de acreditação acadêmica, fazendo parte das estratégias aqui discutidas em conflito no campo da Saúde Coletiva. 

sua importância permanece para a sustentação do setor saúde. A normatividade antes associada a uma forte militância pela reforma universalista do setor saúde deslocou-se hoje para a discussão sobre sua sustentabilidade política. Por sua vez, a análise de políticas, que corresponde a uma área difusa, mas ao mesmo tempo pervasiva e abrangente no país, foi se consolidando com o passar do tempo. $\mathrm{O}$ conhecimento técnico e científico aplicado à resolução de problemas é agora reconhecido como necessário, daí o forte apoio do MS à produção crescente de estudos sobre o sistema de saúde. O papel do MS no apoio e financiamento de estudos orientados às políticas, e o da Abrasco no reconhecimento e reafirmação do caráter multidisciplinar e normativo da Saúde Coletiva garantem legitimidade ao campo. Esse processo, inadvertidamente, contribuiu para a consolidação e profissionalização da análise de políticas no Brasil. ${ }^{5}$

\section{Referências}

ASSOCIAÇĀO BRASILEIRA DE SAÚDE COLETIVA. Nota do fórum de coordenadores de programas de pós-graduação em Saúde Coletiva à presidência do CNPq. São Luis, 16-17 nov 2010. Disponível em: http://www.abrasco.org.br/forumsaudecoletiva/biblioteca/index.php. Acesso em: 30 abr 2012.

AROUCA, S. O Dilema Preventivista: contribuição para a compreensão e crítica da medicina preventiva. São Paulo: Unesp, 2003.

BARDACH E. The eight-step path of policy analysis: A handbook for practice. Berkeley, CA: Berkeley Academic Press, 1996.

BELISÁRIO S. Congressos da Abrasco: a expressão de um espaço construído. In: TRINDADE, N.L.; SANTANA, J.P. (Orgs.). Saúde Coletiva como Compromisso: a trajetória da Abrasco. Rio de Janeiro: Fiocruz, 2006. p.45-78.

BRASIL. Ministério da Educação. Coordenação de Aperfeiçoamento e Pessoal de Nível Superior. Plano Nacional de Pós-Graduação: PNPG 2010-2020. Brasília: Capes, 2010.

CAMARGO JR, K.R. et al. Produção Intelectual em Saúde Coletiva: epistemologia e evidências de diferentes tradições. Revista de Saúde Pública, v.44, n.3, p.394-398, 2010.

COSTA, N.R. A avaliação da produção intelectual e o declínio da interdisciplinariedade na Saúde Coletiva. Physis: Revista de Saúde Coletiva, Rio de Janeiro, v.22, n.2, p.681-699, 2012. Expert community and sectoral policy: the Brazilian Sanitary Reform. In: VAitsman, J.; RIBEIRO, J.M.; LOBATO, L. (Eds.). Policy Analysis in Brazil. Bristol: Policy Press, 2013. p.215-228. 
ESCOREL, S. Reviravolta na Saúde: origem e articulação do movimento sanitário. Rio de Janeiro: Fiocruz, 1998.

FARR, J.; HACKER, J.; KAZEE, N. The Policy Scientist of Democracy: the discipline of Harold Laswell, American Political Science Review, v.100, n.4, p.579-587, Nov 2006.

GEVA-MAY, I. Cultural Theory: the neglected variable in the craft of policy analysis. Journal of Comparative Policy Analysis: Research and Practice, v.4, p.243-265, 2002.

GOLDBAUM, M.; BARATA, R.B. O feito por fazer. TRINDADE, N.L.; SANTANA, J.P. (Orgs.). Saúde Coletiva como Compromisso: a trajetória da Abrasco. Rio de Janeiro: Fiocruz, 2006. p.79-115.

HOLlANDA, E.; SIQUEIRA, S.; STROZENBERG, L. A Formação em Políticas Públicas no Brasil. Relatório Parcial de Pesquisa. Rio de Janeiro: Escola Nacional de Saúde Pública, 2012. HOWLETT, M.; LAYCOCK, P. Studying Public Policy Cycles \& Policy Subsystems. Oxford: Oxford University Press, 2005.

KUHN, T.S. The structure of Scientific Revolutions. Chicago: University of Chicago Press, 1962. LASSWELL, H. The Policy Orientation. In: LERNER, D.; LASSWELL, H. The Policy Sciences. Stanford, CA: Stanford University Press, 1951.

LOYOLA, M.A. A Saga das Ciências Sociais na Área da Saúde Coletiva: elementos para reflexão. Physis: Revista de Saúde Coletiva. Rio de Janeiro, v.18, n.2, p.251-275, 2008.

LUZ, M.T. Prometeu acorrentado: análise sociológica da categoria produtividade e as condições atuais da vida acadêmica. Physis, Revista de Saúde Coletiva, Rio de Janeiro, v.15, n.1, p.39-57, 2005.

McCOOL, D. Public Policy Theories, Models and Concepts: an Anthology. New Jersey: Prentice-Hall, 1995.

NUNES, E. Pós-Graduação em Saúde Coletiva no Brasil: Histórico e Perspectivas. Physis, Rev. Saúde Coletiva, Rio de Janeiro, v.15, n.1, p.13-38, 2005.

PATTON, C.; SAWICKI, D. Basic methods of policy analysis and planning. Englewood Cliffs, NJ: Prentice Hall, 1993.

PELLEGRINI FILHO, A. Pesquisa em saúde, política de saúde e equidade na América Latina. Ciência e Saúde Coletiva, v.9, n.2, p.339-350, 2004.

PIERSON, P. Politics in time: History, Institutions and Social Analysis. Princeton: Princeton University Press, 2004.

TEIXEIRA, S. M. F. As Ciências Sociais em Saúde no Brasil. In: NUNES, E.D. (Org.). As Ciências Sociais em Saúde na América Latina: tendências e perspectivas. Brasília: OPAS, 1985. VAITSMAN, J.; LOBATO, L.; ANDRADE, G. Professionalization of policy analysis in Brazil. In: VAITSMAN, J.; RIBEIRO, J.M.; LOBATO, L. Policy Analysis in Brazil. Policy Press: Bristol, 2013. p.13-26. 
WEIMER D. Policy analysis and evidence: A craft perspective. Policy Studies Journal, v.26, p.114-28, 1998.

WILDAVSKY, A. Speaking Truth to Power. Toronto: Little, Brown and Company, 1979.

\section{Notas}

${ }^{1}$ A Coordenação de Aperfeiçoamento de Pessoal de Nível Superior (CAPES) e o Conselho Nacional de Desenvolvimento Científico e Tecnológico ( $\mathrm{CNPq})$ não são as únicas nesta esfera de governo, na medida em que agências como a FINEP, diversas universidades federais e empresas estatais efetuam investimentos importantes. No entanto, detêm posição formal de incentivar e regular atividades de ensino e pesquisa avançada no país e possuem status de principais ordenadores destas atividades no país.

${ }^{2}$ Valores referentes a instituições apenas do governo federal, excluindo-se os gastos com pessoal ativo e inativo. Não consideramos aqui os gastos significativos dos governos estaduais, afora os gastos de agências e empresas públicas federais citadas como a Finep, a Embrapa (pesquisa agropecuária), Petrobras (pesquisa geofísica) e Ipea (pesquisa social).

${ }^{3}$ Excluídos os gastos com pessoal ativo e inativo destas instituições.

${ }^{4}$ Não existem informações com detalhamento suficiente sobre os programas financiados pelo MS de modo a analisar os desenhos metodológicos que sustentam as pesquisas. No entanto, conforme os objetivos dos programas e ações e das áreas onde estavam alocados, obtivemos uma estimativa dos gastos que atribuímos ao grupo de análise de políticas e treinamento. Foram analisados relatórios de gestão de cada secretaria segundo o último ano disponível. Esses relatórios constituem prestação de contas a órgãos reguladores e apresentam a descrição de gastos e atividades realizadas segundo cada programa de despesa. Consideramos, para fins de simplificação, que os gastos programáticos e as séries históricas de pagamentos e transferências teriam pouca variação no período 2005-2009, embora este erro deva ser considerado na análise. Não espelham o gasto orçamentário global do MS (que inclui despesas administrativas, de pessoal, previdenciárias, dívidas, entre outras).

${ }^{5}$ J. Vaitsman participou da concepção, coleta e análise dos dados, elaboração e finalização do artigo. J.M. Ribeiro participou da concepção, coleta e análise dos dados quantitativos e elaboração do artigo. L.V. Lobato participou da concepção, análise dos dados e elaboração do artigo. 
Policy analysis, health policies and the field of Collective Health

The paper discusses the development in Brazil of the field of Collective Health field as grounded on three principles similar to those of the policy sciences proposed in the US by H. Laswell in the 1950's: multidisciplinarity, resolution of problems and normativity. Since then, the idea of science applied to the improvement of the policy process spread under the name of policy analysis. While in the USA normativity was seen by political scientists as incompatible with science's core values, in Brazil this convergence originated the academic field of collective health in the 1970's when health reform movement, supported by political parties, labor unions and social movements, developed. With democratic regime and deep changes in the health sector, the use of scientific and analytical knowledge by decision makers got momentum in Brazil as well as the role of the collective health which joined knowledge production and political action. The paper shows that as the field developed, a double process occurred: on one hand, the support for policy oriented studies as well as of Abrasco in the assertion of the multidisciplinary and normative features of the collective health. On the other hand, in the sphere of academic accreditation, the epistemic disputes for scientific legitimating and the predominance of academic legitimacy based on biomedicine criteria affecting the access to financial resources and calling into question the normative and multidisciplinary principles that originated the field.

Key words: policy analysis; health policies; Collective Health; multidisciplinarity. 